\title{
Electronic and Magnetic States in the Giant Magneto-resistive Compounds
}

\author{
C. M. Varma \\ ATET Bell Laboratories \\ Murray Hill, NJ 07974
}

\begin{abstract}
The paramagnetic insulator to ferromagnetic metal transition in Lanthanum manganites and the associated magnetoresistive phenomena is treated by considering the localization due to random hopping induced by slowly fluctuating spin configurations and electron-electron interactions. The transition temperature and its variation with composition is derived. The primary effect of the magnetic field on transport is to alter the localization length, an effect which is enhanced as the magnetic susceptibility increases. Expressions for the conductivity, its variation with magnetic field, and its connection with magnetic susceptibility in the paramagnetic phase are given and can be tested with further experiments.
\end{abstract}




\section{INTRODUCTION}

Interest in mixed-valent compounds of the form $\mathrm{A}_{1-\mathrm{x}}^{3+} \mathrm{B}_{\mathrm{x}}^{2+} \mathrm{Mn}_{\mathrm{x}}^{3+} \mathrm{Mn}_{1-\mathrm{x}}^{4+} \mathrm{O}_{3}$, whose fascinating properties were discovered ${ }^{1}$ about fifty year ago, has revived recently. The phase diagram as a function of $\mathrm{x}$ for various trivalent atoms $\mathrm{A}$ and divalent atoms $\mathrm{B}$ and the magneto-transport properties has been determined. ${ }^{2,3}$ For $0.1 \lesssim \mathrm{x} \lesssim 0.3$ an insulating or very high resistance phase at high temperatures is followed at lower temperatures with a metallic phase accompanied by ferromagnetic order. The basic physical point, ${ }^{4}$ made by Zener in 1951, is that the elementary electron conduction process, given that valence states other than $3^{+}$and $4^{+}$are disallowed due to strong local correlations, in which two Mn ions of different valence interchange their valence states: $\mathrm{Mn}^{3+} \mathrm{Mn}^{4+} \rightarrow \mathrm{Mn}^{4+} \mathrm{Mn}^{3+}$, is proportional to the one-electron transfer integral t only when the initial and final states are degenerate. This requires that the Hund's rule coupled spins of $\mathrm{Mn}^{3+}(\mathbf{S}=3 / 2)$ and of $\mathrm{Mn}^{4+}(\mathbf{S}=2)$ are aligned. Otherwise, the transfer integral is of $\mathrm{O}\left(\mathrm{t}^{2} / \mathrm{J}\right)$ where $\mathrm{J}$ is the Hund's rule coupling energy, which for $\mathrm{Mn}^{3+}$ is several $\mathrm{eV}$ and assumed much larger than t. An appropriate model Hamiltonian is

$$
\mathrm{H}=\mathrm{t} \sum_{\langle\mathrm{ij}\rangle}^{\prime} \mathrm{c}_{\mathrm{i} \alpha}^{+} \mathrm{c}_{\mathrm{j} \alpha}+\mathrm{J} \sum_{\mathrm{i}} \mathrm{S}_{\mathrm{i}} \cdot \mathrm{c}_{\mathrm{i}}^{+} \boldsymbol{\sigma} \mathrm{c}_{\mathrm{i}}-\mu \sum_{\mathrm{i}} \mathrm{n}_{\mathrm{i}}
$$

where the electrons hop only between nearest neighbor sites of different valences. For $|J| \gg t$ the spin of the 'conduction' electron is always parallel to the local $\mathrm{S}_{\mathrm{i}}$. Projecting to such states

$$
\mathrm{H}_{\mathrm{eff}}=\sum_{\mathrm{i}, \mathrm{j}} \mathrm{t}\left(\mathbf{S}_{\mathrm{i}},\left(\mathbf{S}_{\mathrm{j}}+\boldsymbol{\sigma}\right)_{\max }\right) \mathrm{c}_{\mathrm{i}}^{+} \mathrm{c}_{\mathrm{j}}
$$

i.e. the transfer integral is a function of the relative orientation of the $\mathrm{Mn}^{4+}\left(\mathrm{S}_{\mathrm{i}}=3 / 2\right)$ spin and $\mathrm{Mn}^{3+}(\mathrm{S}=2)$, i.e. $\left(\mathrm{S}_{\mathrm{j}}+\sigma\right)_{\max }$.

In a pair-wise hopping process the conserved value of the spin is $\left|\mathbf{S}_{\mathrm{i}}+\mathbf{S}_{\mathrm{j}}+\boldsymbol{\sigma}\right|$, so we expect the hopping to depend on this function. Semiclassically the angle $\theta$ between two spins $\mathbf{S}_{1}$ and $\mathbf{S}_{2}$ is given by

$$
\cos \frac{\theta}{2}=\frac{\left|\mathbf{S}_{1}+\mathbf{S}_{2}\right|}{2 \mathrm{~S}}
$$

so that semiclassically the effective hopping Hamiltonian is 


$$
\mathrm{H}_{\mathrm{eff}}=\sum_{(\mathrm{i}, \mathrm{j})}^{\prime} \mathrm{t} \cos \frac{\theta_{\mathrm{ij}}}{2} \mathrm{c}_{\mathrm{i}}^{+} \mathrm{c}_{\mathrm{j}} .
$$

This result was first derived by Anderson and Hasegawa. ${ }^{5}$ If one considers pairwise hopping only, one can specify the relative orientation by $\theta_{\mathrm{ij}}$ alone. More generally, ${ }^{6}$ one must introduce also the azimuthal angle $\phi_{\mathrm{ij}}$ to specify the relative orientations of spins at $\mathrm{i}$ and $\mathrm{j}$ so that a more appropriate form is

$$
\mathrm{H}_{\mathrm{eff}}=\sum_{(\mathrm{i}, \mathrm{j})}^{\prime} \mathrm{t} \mathrm{e}^{\mathrm{i} \phi_{\mathrm{ij}}} \cos \frac{\theta_{\mathrm{ij}}}{2} \mathrm{c}_{\mathrm{i}}^{+} \mathrm{c}_{\mathrm{j}} .
$$

$\phi_{\mathrm{ij}}$ is a Berry phase. This may have some interesting consequences which we hope to discuss in the future.

Since ferromagnetism is accompanied in these materials by metallicity, one may expect that magnetic polarization by an external field will cause a large drop in resistivity. The discovery $^{2,3}$ of such a large magneto-resistance has led to much interest in these materials.

In the next section, I describe how an adiabatic approximation may be introduced by first freezing the spin configuration and calculating the electronic states. It is argued that the chemical potential passes from a region of localized states to delocalized states as $\mathrm{x}$ is increased. Spin polaron effects as correction to the adiabatic approximation are considered next as are electron-electron interaction effects which are expected to produce a gap in the excitation spectra and in one-electron states near the chemical potential. In Section III I calculate the variation of the ferromagnetic transition temperature with $\mathrm{x}$.

\section{GENERAL CONSIDERATIONS}

I am primarily interested in the paramagnetic regime where the spins $\left\{\mathrm{S}_{\mathrm{i}}\right\}$ are randomly oriented and fluctuate at frequencies related only to the temperature $\mathrm{k}_{\mathrm{B}} \mathrm{T}$. Any spin-spin correlation only reduces the characteristic frequency. Further assume that $\mathrm{k}_{\mathrm{B}} \mathrm{T} \ll \mathrm{t}$. Then the core-spin fluctuations may be treated in the adiabatic approximation, in which we first freeze the spins in a random configuration $\left\{\mathbf{S}_{\mathrm{i}}\right\}$ and calculate the electronic states $\psi_{\mathrm{n}}\left(\left\{\mathrm{S}_{\mathrm{i}}\right\}\right)$ in the random configuration. We can then perturbatively couple the thermal fluctuations of spins to find corrections to the electronic wave functions. Simultaneously the potential energy of the spin configurations is given by $\sum_{n} E_{n}\left(\left\{S_{i}\right\}\right) f\left(E_{n}\right)$ where 
$\mathrm{f}$ is the Fermi function. This procedure is quite analogous to the Born-Oppenheimer approximation in electron-phonon problems, except that no independent inertia exists for the spins. One can also investigate the paramagnetic to ferromagnetic transition by this method.

Consider then first a random configuration of spins. The transfer integrals for conduction electrons are then random variables varying from 0 to $|\mathrm{t}|$. The distribution of the transfer integral will be derived in Section III below. This randomness was treated recently $^{7}$ in a dynamic generalization ${ }^{8}$ of the coherent potential approximation (CPA) to calculate the equilibrium and transport properties. In another paper ${ }^{9}$ it was treated perturbatively to calculate the resistivity with the conclusion that the insulating behavior in the paramagnetic phase is inexplicable in purely electronic models and suggesting electron-phonon interactions. This line of thinking has led to the suggestion ${ }^{10}$ that a Jahn-Teller distortion is responsible for the behavior. The phenomena observed in the manganites occurs also in mixed-valent $\mathrm{TmSe}_{\mathrm{x}} \mathrm{Te}_{1-\mathrm{x}}$ compounds. ${ }^{11}$ No question of JahnTeller distortion arises there.

$\mathrm{LaMnO}_{3}$ is an insulator in which the $\mathrm{Mn}-\mathrm{O}_{6}$ octahedra is Jahn-Teller distorted. I think it is not correct to conclude that the insulating behavior is due to the Jahn-Teller distortion. Most transition metal perovskite compounds are insulators, even $\mathrm{LaTiO}_{3}$ where effective electron-electron interactions on $\mathrm{Ti}$ are smaller than in $\mathrm{Mn}$ due to more effective S-electron screening. These are all Mott insulators. Note that the derivation of the double exchange interaction assumes correctly that the direct electron-electron interaction is even larger than the exchange interaction J. If symmetry allows a JahnTeller distortion in the insulating phase, such a distortion parasitically does occur. The conditions for Jahn-Teller effects in metals are much more stringent and in any case not required by symmetry and not expected to lead to insulating phase when the metallic bandwidth is $\mathrm{O}(2 \mathrm{eV})$. One expects that the Jahn-Teller distortion of $\mathrm{LaMnO}_{3}$ will appear in reduced magnitudes even on addition of $\mathrm{Sr}$ in the insulating phase and decrease in the low temperature metallic phase. More important than the Jahn-Teller distortions for quantitative purposes are the breathing mode polaronic distortions which are different around $\mathrm{Mn}^{3+}$ and $\mathrm{Mn}^{4+}$ because of the large difference in ionic size. 
In a model for random hopping, one expects electronic states to be localized at least for some energies. CPA is a (self-consistent) single-site approximation while localization arises from interference among scatters. While the CPA is a very good approximation for many purposes, it cannot capture the physics of localization. I believe the transport properties in this paramagnetic regime for most of the range of composition as well as the effect of magnetic field on them can be understood only when the localization of the single particle states is considered.

The present problem is one of off-diagonal randomness as opposed to the Anderson model $^{12}$ for disorder. This has not been so thoroughly investigated. One approximate but reliable calculation of the nature of the wave functions in models of off-diagonal randomness is due to Economu and Antoniu. ${ }^{13}$ Their results are sketched in fig. (1). For a model in which the hopping matrix elements $t_{i j}$ are randomly distributed over a semi-circular distribution with mean $t_{0}$ and edges at $t_{0} \mp t_{1}$ they find that for $t_{1} \approx t_{0}$, which is relevant for our case, states in the energy region $\frac{1}{2}|\mathrm{~W}| \lesssim \mathrm{E} \lesssim|\mathrm{W}|$ are localized while these in the middle are extended. $\mathrm{W}$ is the bandwidth of the bulk which CPA gives correctly as about 0.7 of the total bandwidth.

The localization length in a problem of off-diagonal disorder is expected to diverge both at the band-edges and at the mobility edges. The latter is familiar, and as in models of diagonal disorder. The former arises because states at the band-edges are the exponentially rare states which travel through the crystal through routes with identical hopping.

Unlike Ref. (13) the distribution of disorder in the present problem is not symmetrical. It follows from eq. (14) below that the distribution $\mathrm{P}(\mathrm{t})$ increases linearly with $\mathrm{t}$ with a cutoff at the maximum value. The higher moments of the distribution are therefore larger than in Ref. (13). So the region of localized states is expected to be larger than in Fig. (1). Moreover strong correlations in the present problem (in the presence of disorder) further favor localization. The effective fluctuations in $t$ are further increased by different polaronic renormalizations of $\mathrm{t}$ and $\mathrm{Mn}^{3+}$ ion and an $\mathrm{Mn}^{4+}$ ion.

We may safely assume that in the frozen spin approximation, states at the chemical potential are localized for low dopings and that a mobility edge occurs so that for higher 
dopings the states at the chemical potential are delocalized although strongly scattering. CPA should be a good approximation to calculate properties in the second regime.

\section{Spin-polaron Formation: First Correction to the Adiabatic Approximation}

The spins do not have any inertia. The leading correction to the adiabatic approximation then amounts to (i) minimizing the free-energy $\mathrm{F}\left(\left\{\mathrm{S}_{\mathrm{i}}\right\}\right)$ with respect to spin configurations $\left\{\mathrm{S}_{\mathrm{i}}\right\}$ and (ii) calculating scattering between electronic states due to fluctuations about the new configuration. The first part is the same as considering spin-polaron formation.

We can easily make the first correction to the frozen random spin-configuration by

the formation of spin polaron around an electronic state which otherwise would be highly localized. Let there be $\mathrm{P}$ lattice sites which are spin-polarized so that an electron can hop freely between them to lower its kinetic energy. This is opposed by the entropy lost by the spins. So $\mathrm{P}$ is determined by minimizing

$$
\mathrm{E}(\mathrm{P})=-\mathrm{W}+\frac{\mathrm{at}_{0}}{\mathrm{P}^{2 / 3}}+\mathrm{PkT} \ln (2 \mathrm{~S}+1)
$$

where a is a numerical factor of $\mathrm{O}(1)$, so that

$$
\mathrm{P} \approx\left(\mathrm{at}_{0} / \mathrm{T}\right)^{3 / 5}
$$

This is an elementary generalization to finite temperature of Nagaoka's result ${ }^{14}$ for such models that, at $\mathrm{T}=0$, a single carrier will induce ferromagnetic order. In the manganites the effective $t$ (renormalized for effect other than spin fluctuations, phonons for instance) is about $0.2 \mathrm{eV}$. So at room temperatures the size of the spin-polaron is only a few bonds. So the spin-polaron effect appears not to invalidate the adiabatic approximations over the bulk of the band in the paramagnetic range of temperatures of interest.

A prediction following from these considerations is that the effective moment in susceptibility measurements even at fairly high temperatures will be larger than that of the appropriate average of $\mathrm{Mn}^{3+}$ and $\mathrm{Mn}^{4+}$ moments. At a concentration $\mathrm{x}$ of the spin $\mathrm{S}_{1}$ and the rest $\mathrm{S}_{2}$, one expects

$$
\mathrm{S}_{\text {eff }}^{2}=\mathrm{x}\left(\mathrm{S}_{1}+\mathrm{PS}_{2}\right)\left(\mathrm{S}_{1}+\mathrm{PS}_{2}+1\right)+(1-\mathrm{x}-\mathrm{Px}) \mathrm{S}_{2}\left(\mathrm{~S}_{2}+1\right)
$$


The estimate of spin-polaron effects would be quite altered for states in the tails of Fig. (1) or those near the mobility edge which have a large localization length. The polaron effects would have a major effect only on the surface of such states leaving the bulk of it relatively unaffected. So Eq. (8) is an overestimate. Moreover (8) is merely the first sign of fluctuations above $\mathrm{T}_{\mathrm{c}}$ which increase the susceptibility over the Curie-law.

The adiabatic assumption made here is accompanied by the ergodic hypothesis. The spin-configurations change locally changing the localization length of the electronic state in a given small region. But averaged over the sample the distribution of the energies and localization lengths of the states stays the same. This process does not invalidate the adiabatic approximation unless the temperature is so high that several states of energy $\lesssim 0(\mathrm{kT})$ larger than the chemical potential have spatial overlap with states at the chemical potential. This stability argument gets stronger due to the role of electronelectron interactions in depleting states near the chemical potential discussed below.

The last paragraph is only a plausibility argument. It is a very interesting unsolved theoretical problem to ask for the frequency scale up to which disorder can vary without delocalizing states.

\section{Electron-electron Interactions}

The role of electron-electron interactions is always more important for localized states where the kinetic energy has been quenched than for Bloch states. Efros and Shklovskii ${ }^{15}$ have given convincing arguments that Coulomb interactions create a pseudo-gap at the chemical potential if it lies below the mobility edge. Therefore due to electron-electron interactions the density of one-particle states is modified from Fig. (1) to that depicted in Fig. (2). In three dimensions, the density of states in the localized regime per unit volume near the chemical potential is of the form

$$
\begin{aligned}
\mathrm{g}(\epsilon) & =\epsilon^{2} / \Delta^{3} \\
\Delta & =\alpha \frac{\mathrm{e}^{2}}{\kappa}
\end{aligned}
$$

where $\alpha$ is a numerical constant and $\kappa$ is the dielectric constant. $g(\epsilon)$ is independent of the localization length near the chemical potential. Equation (9) is arrived at by 
considering stability of one particle excitations under excitonic renormalizations due to the Coulomb interactions. Corrections to it due to multiparticle excitations have not yet been established conclusively.

The best way to experimentally test the localization idea and (9) is a tunneling measurement. Density of states of the form (9) have however already been observed in photoemission experiments. ${ }^{16,17}$ The key point is that the density of states is zero at the chemical potential (with $\mathrm{T} / \Delta$ corrections) independent of doping if the chemical potential is below the mobility edge and the experiment is done in the paramagnetic regime.

\section{Resistivity}

The conductivity of the localized states is expected to be of the variable range hopping form:

$$
\sigma \sim \exp \left[-\left(\mathrm{T}_{0} / \mathrm{T}\right)^{1 / 2}\right]
$$

with $\mathrm{T}_{0} \approx \mathrm{e}^{2} / \kappa \ell$, for $\mathrm{T} \ll \mathrm{T}_{0}$. Here $\ell$ is the localization length for states near the chemical potential. As the effective disorder is decreased by applying a magnetic field, $\ell$ increases leading to a decrease in the resistivity. The relationship of the effective disorder and the magnetization is derived in the next section. In the paramagnetic regime, the leading dependence of the localization length on $\mathrm{H}$ is

$$
\ell(\mathrm{H})=\ell_{0}\left(1+\chi(\mathrm{T}) \mathrm{H}^{2} / \overline{\mathrm{t}}\right)
$$

where $\ell_{0}$ depends on the electron density and $\overline{\mathrm{t}}$ is $\mathrm{O}(\mathrm{t})$. Equation (10) may be combined with Eq. (11) to suggest that magnetoresistance plotted against the magnetic susceptibility should fall on the same curve for different temperatures and for different compositions. Similar scaling behavior should be observed for other transport properties as well.

Note that the leading temperature dependence of the conductivity (or magnetoconductivity) exhibited in Eq. (10) gives the number of carriers participating in the conduction. Their mobility is a weakly temperature (and field) dependent pre-factor of (10). This is consistent with the recent observations of Ong et al. ${ }^{18}$ from Hall effect 
and magnetoresistance measurements that the dominant effect of magnetic fields is to increase the number of carriers.

The frequency dependent conductivity should exhibit the effects of the EfrosShklovskii gap in the paramagnetic phase. A gap is indeed observed. ${ }^{19}$ The low energy $\sigma(\omega)$ appears roughly $\sim \omega$. This is quite remarkable and needs more detailed consideration.

When $T_{0} \sim T$, the conductivity is no longer of the form (10). Now several excited states of energy $\leq 0(\mathrm{kT})$ overlap the states at the chemical potential. The mobility may now be calculated from

$$
\mu=\frac{\mathrm{eD}}{\mathrm{kT}}
$$

where the diffusion constant $\mathrm{D} \approx \ell^{2} / \tau ; \ell$ is the typical distance between states degenerate to within $\mathrm{kT}$ which at very high temperatures is $0(\mathrm{a})$ the lattice constant; and $1 / \tau$ is the typical frequency of spin fluctuation $\sim 0(\mathrm{kT})$. So the high temperature conductivity approaches a constant proportional to the carrier density.

\section{ESTIMATE OF METALLIC/FERROMAGNETIC TRANSITION TEMPERATURE}

We can calculate the equilibrium properties in the paramagnetic phase and an estimate of the ferromagnetic transition temperature $\mathrm{T}_{\mathrm{c}}$ by a variational calculation of the free-energy. Let the probability distribution of the angle between neighboring spins $\theta$ be given by

$$
\mathrm{P}(\mathrm{x})=\mathrm{M}^{2} \delta(1-\mathrm{x})+\left(1-\mathrm{M}^{2}\right) \mathcal{N} \exp \left[-\frac{\mathrm{A}}{\mathrm{kT}}(1-\mathrm{x})\right]
$$

where $\mathrm{x}=\cos \theta, \mathrm{M}$ is the uniform magnetization which is finite for $\mathrm{T}>\mathrm{T}_{\mathrm{c}}$ only if a magnetic field $\mathrm{H}$ is applied, $\mathrm{A}$ is a variational parameter which is a function of temperature and $\mathcal{N}$ is a normalization factor. $\mathrm{P}(\cos \theta)$ for $\mathrm{A} / \mathrm{kT} \rightarrow \infty$ aligns all the spins while for $\mathrm{A} / \mathrm{kT} \rightarrow 0$, the spins are completely randomly oriented provided $\mathrm{M}^{2}=0$. So it is expected that as temperature decreases the variational calculation will lead to $\mathrm{A}(\mathrm{T}) / \mathrm{kT}$ changing from 0 to very large values near the ferromagnetic transition. To calculate the 
energy of the electrons, we evaluate the probability distribution of the nearest neighbor transfer integral $\mathrm{P}(\mathrm{t})$ :

$$
\begin{aligned}
\mathrm{P}(\mathrm{t}) & =\frac{1}{\mathrm{t}_{0}} \int_{-1}^{1} \mathrm{~d}(\cos \theta) \mathrm{P}(\cos \theta) \delta\left(\mathrm{t} / \mathrm{t}_{0}-\cos \theta / 2\right) \\
& =\mathrm{M}^{2} \delta\left(\mathrm{t}-\mathrm{t}_{0}\right)+\left(1-\mathrm{M}^{2}\right) \mathcal{N} \frac{\mathrm{t}}{\mathrm{t}_{0}^{2}} \exp \left[-\frac{2 \mathrm{~A}}{\mathrm{kT}}\left(\frac{\mathrm{t}^{2}}{\mathrm{t}_{0}^{2}}-1\right)\right]
\end{aligned}
$$

One should now calculate the electronic density of states $\mathrm{n}(\mathrm{E})$ with $\mathrm{P}(\mathrm{t})$ and calculate the electronic energy in terms of $n(E)$. $n(E)$ can not be evaluated without a lengthy numerical calculation. We will instead use the following approximate method: For a rectangular density of states with bandwidth $\mathrm{W}$, with height $\mathrm{W}^{-1}$ so that it can accommodate at most one electron per atom, the energy at $\mathrm{T}=0$ for $\mathrm{c}$ electrons per atom is

$$
\epsilon(\mathrm{t})=-\frac{\mathrm{W}}{2} \mathrm{c}(1-\mathrm{c})
$$

A good approximation for the electronic energy is

$$
\mathrm{E}_{\mathrm{e}}=\int \mathrm{dt} \mathrm{P}(\mathrm{t}) \epsilon(\mathrm{t})
$$

Classical approximation to the entropy of spins in the large S limit using distribution such as $\mathrm{P}(\mathrm{x})$ give incorrect answers at high temperatures. To calculate the entropy we write the quantum version of $\mathrm{P}(\mathrm{x})$ by writing

$$
\cos \theta-1=\frac{\mathrm{J}^{2}-4 \mathrm{~S}^{2}}{2 \mathrm{~S}^{2}}
$$

where $\mathrm{S}$ is the value of the spin of an ion $\left(\mathrm{Mn}^{3+}\right.$ spin $\mathrm{S}=2$ is the appropriate value) and $\mathrm{J}$ ranges from 0 to $2 \mathrm{~S}$ in integer increments. Then define the quantum analog of the second tern of $(1)$

$$
\mathrm{Z}=\sum_{\mathrm{J}} \mathrm{e}^{-\frac{\mathrm{A}}{\mathrm{kT}} \mathrm{S}^{2}\left(\mathrm{~J}^{2}-4 \mathrm{~S}^{2}\right)}
$$

and

$$
\mathrm{S}_{\mathrm{ions}}=-\frac{\partial}{\partial \mathrm{T}}(\mathrm{kT} \ln \mathrm{Z})
$$

Strictly speaking I should use (18) for the calculation of the energy also, but the final answer is likely to be nearly the same and the form of $\mathrm{P}(\mathrm{x})$, equation (13) leads to $\mathrm{P}(\mathrm{t})$ of Eq. (14) which seems physically more transparent. 
In my calculations I have neglected the entropy of the orbital motion of the $\mathrm{x} /$ unit cell "conduction" electrons compared to that of the $1 /$ unit cell spins. This is a small correction. Similarly finite temperature correction to (3) were neglected.

Free energy then is

$$
\mathrm{F} \approx \mathrm{E}_{\mathrm{e}}-\mathrm{T} \mathrm{S}_{\mathrm{spins}}-\mathbf{M} \cdot \mathbf{H}
$$

We may write this as

$$
\mathrm{F}=\mathrm{t}_{0}\left[\alpha\left(\mathrm{A}, \mathrm{T} / \mathrm{t}_{0}\right) \mathbf{M}^{2}+\mathrm{F}_{0}\left(\mathrm{~A}^{2}, \mathrm{~T} / \mathrm{t}_{0}\right)\right]-\mathbf{M} \cdot \mathbf{H}
$$

so that the uniform magnetic susceptibility is given by

$$
\chi^{-1}=2 \mathrm{t}_{0} \alpha\left(\mathrm{A}, \mathrm{T} / \mathrm{t}_{0}\right)
$$

The ferromagnetic transition temperature is given by

$$
\alpha\left(\mathrm{A}^{2}, \mathrm{~T}_{\mathrm{c}} / \mathrm{t}_{0}\right)=0
$$

The result of the minimization of the free-energy with respect to A, not surprisingly (since this is a mean-field calculation), is that $\chi(\mathrm{T})$ follows a Curie law

$$
\chi(\mathrm{T}) \approx \frac{\mathrm{S}^{2}}{\mathrm{~T}-\mathrm{T}_{\mathrm{c}}}
$$

The numerical minimization of $\mathrm{F}$ with respect to $\mathrm{A}$ gives that

$$
\mathrm{k}_{\mathrm{B}} \mathrm{T}_{\mathrm{c}} \simeq 0.1 \mathrm{E}_{\mathrm{coh}}^{\mathrm{F}}(\mathrm{c})
$$

where $\mathrm{E}_{\mathrm{coh}}^{\mathrm{F}}(\mathrm{c})$ is the electronic cohesive energy for the ferromagnetic case

$$
\mathrm{E}_{\mathrm{coh}}^{\mathrm{F}}(\mathrm{x})=\frac{\mathrm{W}}{2} \mathrm{c}(1-\mathrm{c})
$$

The numerical factor of $\sim 0.1$ reflects (i) that the electronic cohesive energy increases by only about $20 \%$ in going from the complete random spin-orientation to the ferromagnetic configuration and (ii) that the entropic free energy of spins at a temperature $\mathrm{T}=\mathrm{W}$ is about a factor of 2 larger than $\mathrm{W}$.

Mattheiss has calculated the conduction electron bandwidth in the local density approximation to $\mathrm{be} \approx 2.5 \mathrm{eV}$. Then at $\mathrm{x} \approx 0.3$, the calculated $\mathrm{T}_{\mathrm{c}} \approx 250$ which, considering 
the crudeness of the calculation, is in the right range. A more important test is the relation $\mathrm{T}_{\mathrm{c}} \sim \mathrm{x}(1-\mathrm{x})$ which is compared with experimental results in Fig. (2).

CPA is a perfectly respectable approximation to calculate the energetics. Therefore Furukawa's calculations ${ }^{7}$ for $T_{c}$ are probably better than the estimates here although the considerations here may be more transparent.

\section{CONCLUDING REMARKS}

An attempt has been made to understand the equilibrium and transport properties of mixed-valent magnetic compounds on the basis of electronic localization due to magnetic disorder and the alteration of this disorder by a magnetic field. A paramagnetic insulator to Ferromagnetic transition in the observed range of temperatures and with the observed composition dependence is derived. The primary effect of a magnetic field on transport is to alter the localization length, an effect which is enhanced as the magnetic susceptibility increases. Scaling of the transport properties with the magnetic susceptibility and tunneling experiments to observe the Efros-Shkloveskii correlation induced pseduo-gap in the paramagnetic phase are suggested.

In this paper the ordinary antiferromagnetic exchange between localized carriers and the effects due to A-B disorder have been ignored. At low temperatures their effects have been adequately treated by deGennes. ${ }^{20}$

\section{Note Added}

Based on a preprint of this note, the authors of Ref. (10) have in yet another paper on the Jahn-Teller distortion idea (A. J. Millis, R. Mueller, B. I. Shraiman, preprint) pointed out in effect that the fluctuations in hopping estimated here using the results of Economu and Antoniu are small by about a factor of 2 to give the mobility edge at $\mathrm{x} \approx 0.3$. This is picayune. The point of this work is to show an important effect which systematically explains observations as $\mathrm{x}$ is varied. Estimates of the fluctuation in hopping based on "one-electron approximation" can easily be in error by a factor of two or more. As pointed

out here electron correlation effects increase the tendency to localization and increase the 
effective disorder parameter. So does the different lattice distortion (breathing mode) around $\mathrm{Mn}^{3+}$ and $\mathrm{Mn}^{4+}$ which renormalizes t. The uncertanity in disorder fluctuations by a factor of 2 should be compared with a general lack of knowlege of coupling constrants in the Jahn Teller scheme. In any case recent neutron scattering results strongly disfavor Jahn Teller effects as responsible for the insulating to metallic crossover (M. Marezio, private communications).

\section{Acknowledgements}

I wish to acknowledge useful discussions with G. Aeppli, B. Batlogg, S. Cheong, H. Hwang, P. B. Littlewood, A. J. Millis, T. Paalstra, A. Ramirez, B. I. Shraiman, Y. Tokura, and especially H. Mathur and A. Sengupta. 


\section{REFERENCES}

1. G. H. Jonker and J. H. van Santen, Physica (Utrecht) 16, 337 (1950); 19, 120 (1953).

2. R. M. Kusters et al., Physics (Amsterdam) 155B, 362 (1989); K. Chahara et al., Appl. Phys. Lett. 63, 1990 (1993); M. McCormack et al., Appl. Phys. Lett. 64, 3045 (1994); G. C. Xiong et al., Solid State Comm. (to be published); Phys. Rev. (to be published); H. Y. Hwang et al., Phys. Rev. Lett. 75, 914 (1995); J. H. Schriffer et al., Phys. Rev. Lett. 75, 3336 (1995).

3. Y. Tokura et al., J. Phys. Soc. Japan 63, 3931 (1994); A. Asamitsu et al., Nature (London) 373, 407 (1995); S. Jin et al., Science 264, 413 (1994).

4. T. Paalstra et al., (Preprint); C. Zener, Phys. Rev. 82, 403 (1951).

5. P. W. Anderson and H. Hasegawa, Phys. Rev. 100, 675 (1955).

6. H. Mathur (Private Communication).

7. N. Furukawa, J. Phys. Soc. Japan 63, 3214 (1994) and Preprints.

8. A. George, G. Kotliar, M. Rozenberg, Rev. Mod. Phys. (in print).

9. A. J. Millis, P. B. Littlewood and B. I. Shraiman, Phys. Rev. Lett. 74, 5144 (1995).

10. A. J. Millis, B. Shraiman and M. Mueller, (preprint).

11. C. M. Varma, Solid State Comm, 30, 537 (1979); B. Batlogg, H. R. Ott, and P. Wachter, Phys. Rev. Lett. 42, 282 (1979).

12. P. W. Anderson, Phys. Rev. 109, 1492 (1961).

13. E. N. Economu and P. D. Antoniu, Sol. State Comm. 21, 285 (1977).

14. Y. Nagaoka, Phys. Rev., 147, 392 (1966). 
15. B. I. Shklovskii and A. L. Efros, Chapter 10 in Electronic Properties of Doped Semiconductors, Springer-Verlag, Berlin (1984).

16. T. Saitoh et al., Phys. Rev. B51, 13942 (1995), and lecture at Naeba Conference, Japan, Oct. 31 (1995).

17. J. H. Park et al., preprint.

18. N. P. Ong, (Private Communication).

19. Y. Okimoto, Phys. Rev. Lett. 75, 109 (1995).

20. P. G. deGennes, Phys. Rev., 118, 141 (1960). 


\section{FIGURE CAPTIONS}

Fig. 1a Character of states as a function of their energy in a tight binding model with disorder in which $t_{0}$ is the mean and $t_{1}$ the rms width of the transfer integral. This sketch is based on Economu and Antoniu, Ref. (13).

Fig. 1b Sketch of the density of states.

Fig. 2 Density of states with Efros-Shklovskii gap.

Fig. 3 Magnetic transition temperature vs $\mathrm{x}$ in $\mathrm{La}_{7-\mathrm{x}} \mathrm{Sr}_{\mathrm{x}} \mathrm{MnO}_{3}$ taken from Ref. (3), compared to the result $\mathrm{T}_{\mathrm{M}} \sim \mathrm{x}(1-\mathrm{x})$ from Eq. (25). 